\title{
Adhesion between Elastic Bodies with Randomly Rough Surfaces
}

\author{
B. N. J. Persson \\ IFF, FZ-Jülich, 52425 Jülich, Germany \\ (Received 5 April 2002; published 21 November 2002)
}

\begin{abstract}
I have developed a theory of adhesion between an elastic solid and a hard randomly rough substrate. The theory takes into account the fact that partial contact may occur between the solids on all length scales. I present numerical results for the case where the substrate surface is self-affine fractal. When the fractal dimension is close to 2 , complete contact typically occurs in the macroasperity contact areas. For a fractal dimension larger than 2.5, the area of (apparent) contact decreases continuously when the magnification is increased.
\end{abstract}

DOI: $10.1103 /$ PhysRevLett.89.245502

PACS numbers: $81.40 . \mathrm{Pq}, 62.20 .-\mathrm{x}$

Even a highly polished surface has surface roughness on many different length scales. When two bodies with nominally flat surfaces are brought into contact, the area of real contact will usually be only a small fraction of the nominal contact area.

How large is the area of real contact between a solid block and the substrate? This fundamental question has extremely important practical implications. For example, it determines the contact resistivity and the heat transfer between the solids. It is also of direct importance for sliding friction [1,2], e.g., the rubber friction between a tire and a road surface, and it has a major influence on the adhesive force between two solid blocks in direct contact. I have developed a theory of contact mechanics [3], valid for randomly rough (e.g., self-affine fractal) surfaces, but neglecting adhesion (see also [4]). In this Letter, we consider adhesion for randomly rough surfaces for the most general case where partial contact occurs at the interface on many different length scales; see Fig. 1.

The influence of surface roughness on the adhesion between rubber (or any other elastic solid) and a hard substrate has been studied in a classic paper by Fuller and Tabor [5] (see also [6-9]). They found that even a relative small surface roughness can remove the adhesion completely. In order to understand the experimental data, they developed a very simple model based on the assumption of surface roughness on a single length scale. However, when roughness occurs on many different length scales, a qualitatively new picture emerges.

The present theory is based on the contact mechanics theory developed in Refs. [3,9]. This theory recognizes that it is essential not to exclude a priori any roughness length scale from the analysis. Thus, if $A(\lambda)$ is the (apparent) area of contact on the length scale $\lambda$ [more accurately, we define $A(\lambda)$ to be the real contact area (projected on the $x y$ plane) if the surface would be smooth on all length scales shorter than $\lambda$ ], then we study the function $P(\zeta)=$ $A(\lambda) / A(L)$, which is the relative fraction of the rubber surface area where contact occurs on the length scale $\lambda=L / \zeta$ (where $\zeta \geq 1$ ), with $P(1)=1$. Here $A(L)=A_{0}$ denotes the macroscopic (nominal) contact area $[L$ is the diameter of the macroscopic contact area so that $\left.A_{0} \approx L^{2}\right]$.

Consider a rubber ball (radius $R_{0}$ ) in adhesive contact with a perfectly smooth and hard substrate. The elastic deformation of the rubber can be determined by minimizing the total energy, which is the sum of the (positive) elastic energy stored in the deformation field in the rubber ball and the (negative) binding energy between the ball and the substrate at the contact interface. The free energy minimization gives the Johnson-Kendall-Roberts pulloff force [10]: $F_{\mathrm{c}}=(3 \pi / 2) R_{0} \Delta \gamma$, where $\Delta \gamma=\gamma_{1}+\gamma_{2}-$ $\gamma_{12}$ is the change in the surface free energy (per unit area) upon contact due to the rubber-substrate interaction. Consider now the same problems as above, but assume that the substrate surface has roughness described by the function $z=h(\mathbf{x})$ and that the width $L$ of the nominal contact area is much larger than the long-distance cutoff

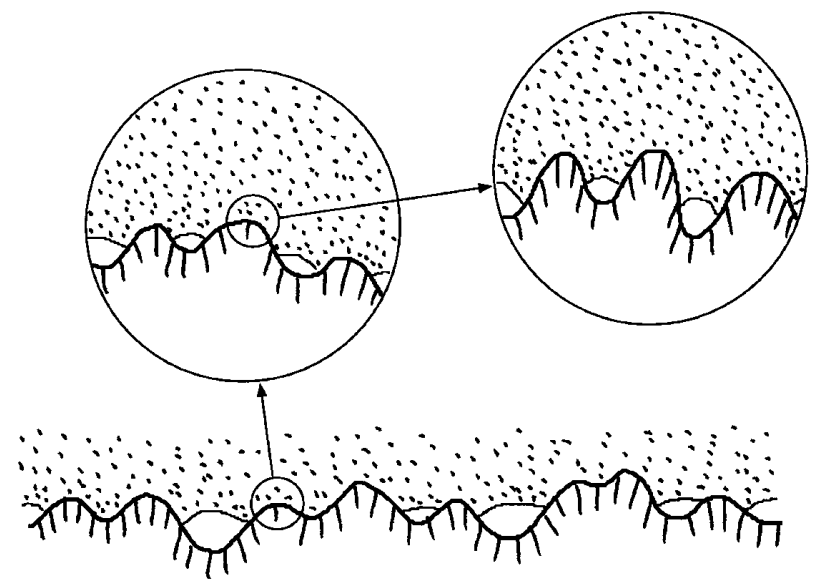

FIG. 1. A rubber block (dotted area) in adhesive contact with a hard rough substrate (dashed area). The substrate has roughness on many different length scales and the rubber makes partial contact with the substrate on all length scales. When a contact area is studied at low magnification it appears as if complete contact occurs, but when the magnification is increased it is observed that, in reality, only partial contact occurs. 
(or rolloff) length $\lambda_{0}$ of the surface roughness power spectra. In this case, we can still use the result for $F_{\mathrm{c}}$ above (even when only partial contact occurs at the interface), but with $\Delta \gamma$ replaced by $\gamma_{\mathrm{eff}}(1)$ given below.

Consider the system at the length scale $\lambda=L / \zeta$, where $L$ is of order the diameter of the nominal contact area. We define $q_{L}=2 \pi / L$ and write $q=q_{L} \zeta$. Let $P(\sigma, \zeta)$ denote the stress distribution in the contact areas under magnification $\zeta$. The function $P(\sigma, \zeta)$ satisfies the differential equation (see Ref. [3]):

$$
\frac{\partial P}{\partial \zeta}=f(\zeta) \frac{\partial^{2} P}{\partial \sigma^{2}}
$$

where

$$
f(\zeta)=\frac{\pi}{4}\left[\frac{E}{\left(1-\nu^{2}\right)}\right]^{2} \zeta^{3} q_{L}^{4} C\left(\zeta q_{L}\right),
$$

where $E$ is the elastic modulus and $\nu$ the Poisson ratio. The surface roughness power spectra

$$
C(q)=\frac{1}{(2 \pi)^{2}} \int d^{2} x\langle h(\mathbf{x}) h(\mathbf{0})\rangle e^{-i \mathbf{q} \cdot \mathbf{x}},
$$

where $z=h(\mathbf{x})$ is the height of the surface above a flat reference plane (chosen so that $\langle h\rangle=0$ ), and $\langle\cdots\rangle$ stands for ensemble average.

The relative (apparent) area of contact (projected on the surface $x y$ plane) when the system is studied under the magnification $\zeta$ can be written (see Ref. [3]):

$$
P(\zeta)=\int_{-\sigma_{\mathrm{a}}}^{\infty} d \sigma P(\sigma, \zeta)=1-\int_{1}^{\zeta} d \zeta^{\prime} S\left(\zeta^{\prime}\right),
$$

where the detachment stress $\sigma_{\mathrm{a}}=\sigma_{\mathrm{a}}(\zeta)$ is defined below. $S(\zeta)$ can be obtained by solving Eq. (1) subjected to the boundary conditions:

$$
P\left[-\sigma_{\mathrm{a}}(\zeta), \zeta\right]=0, \quad P(\infty, \zeta)=0 .
$$

These equations state that detachment occurs when the local stress on the length scale $L / \zeta$ reaches $-\sigma_{\mathrm{a}}(\zeta)$ and that there is no infinitely large stress at the interface.

Let us consider the system on the characteristic length scale $\lambda=L / \zeta$. The quantity $\sigma_{\mathrm{a}}(\zeta)$ is the stress necessary to induce a detached area of width $\lambda$ and can be obtained from standard arguments related to a penny-shaped crack of diameter $\lambda[11]$ :

$$
\sigma_{\mathrm{a}}=\left[\frac{\gamma_{\mathrm{eff}}(\zeta) E q}{2\left(1-\nu^{2}\right)}\right]^{1 / 2},
$$

where $q=2 \pi / \lambda=\zeta q_{L}$. Here $\gamma_{\text {eff }}(\zeta)$, the change in the effective surface energy upon contact, is given by

$$
\begin{aligned}
\frac{\gamma_{\mathrm{eff}}\left(q_{\mathrm{a}}\right)}{\Delta \gamma}= & \frac{P\left(q_{1}\right)}{P\left(q_{\mathrm{a}}\right)} \int_{0}^{\infty} d x\left(1+\xi^{2} x\right)^{1 / 2} e^{-x} \\
& -\frac{2 \pi}{\delta} \int_{q_{\mathrm{a}}}^{q_{1}} d q q^{2} \frac{P(q)}{P\left(q_{\mathrm{a}}\right)} C(q),
\end{aligned}
$$

where

$$
\xi^{2}=\int d^{2} q q^{2} C(q)
$$

and $\delta=4\left(1-\nu^{2}\right) \Delta \gamma / E$. The first term on the right-hand side in Eq. (7) is the contribution from the adhesional interaction (which depends on the area of real contact) [12] and the second term is the elastic energy stored at the interface. In the limiting case $P(\zeta) \equiv 1$ (complete contact), Eq. (7) reduces to the result derived in Ref. [9].

If we assume a constant external pressure $\sigma_{0}$ in the nominal contact area, then $P(\sigma, 1)=\delta\left(\sigma-\sigma_{0}\right)$. From Eqs. (1)-(4) one can show that

$$
\int_{1}^{\zeta} d \zeta^{\prime} S\left(\zeta^{\prime}\right)\left(\frac{a(\zeta)}{a(\zeta)-a\left(\zeta^{\prime}\right)}\right)^{1 / 2} \exp \left(-\frac{\left[\sigma_{\mathrm{a}}(\zeta)-\sigma_{\mathrm{a}}\left(\zeta^{\prime}\right)\right]^{2}}{4\left[a(\zeta)-a\left(\zeta^{\prime}\right)\right]}\right)=\exp \left(-\frac{\left[\sigma_{\mathrm{a}}(\zeta)+\sigma_{0}\right]^{2}}{4 a(\zeta)}\right)
$$

where

$$
a(\zeta)=\int_{1}^{\zeta} d \zeta^{\prime} f\left(\zeta^{\prime}\right)
$$

Equation (9) is a linear integral equation for $S(\zeta)$ which is easy to solve by matrix inversion.

Let us first assume that $\sigma_{\mathrm{a}}(\zeta)$ is independent of $\zeta$. In this case (4) and (9) gives

$$
P(\zeta)=\frac{2}{\pi} \int_{0}^{\infty} d x \frac{\sin x}{x} e^{-x^{2} G}
$$

where $G(\zeta)=a(\zeta) /\left(\sigma_{0}+\sigma_{a}\right)^{2}$. We note that, for small effective loading pressure $\sigma_{0}+\sigma_{\mathrm{a}}$, we have $G \gg 1$ for large enough $\zeta$, and in this case only $x \ll 1$ will contribute to the integral in (11) and we can replace $\sin x \approx x$ to obtain $P(\zeta) \approx[\pi G(\zeta)]^{-1 / 2} \sim \sigma_{0}+\sigma_{\mathrm{a}}$. Thus, in this limit the area of real contact is proportional to the sum of the applied pressure $\sigma_{0}$ and the "adhesion" pressure $\sigma_{\mathrm{a}}$. This result has often been used [13], e.g., in discussing friction, but we point out that it is valid only when $\sigma_{\mathrm{a}}(\zeta)$ is independent of $\zeta$. This will never be the case when surface roughness occurs on many different length scales.

Let us now present numerical results obtained from (4) and (6) $-(10)$ by iteration. We assume that the rough surface is self-affine fractal [14] with the Hurst exponent $H$ corresponding to the fractal dimension $D_{\mathrm{f}}=3-H$. We consider first the case $H=0.8$ or $D_{\mathrm{f}}=2.2$, which is typical for many surfaces of practical interest, e.g., surfaces prepared by fracture or sandblasting, and we assume that the surface is self-affine over two decades in length scales, $q_{0}<q<q_{1}$, where $q_{0}=0.01 q_{1}$. Thus, the maximum magnification $\zeta_{1}=q_{1} / q_{0}=100$ and the area of real contact $A_{0} P\left(\zeta_{1}\right)$ is determined by studying the system at this magnification.

Figure 2 shows (a) the effective interfacial energy $\gamma_{\mathrm{eff}}(1)$ and (b) the normalized area of real contact, $P\left(\zeta_{1}\right)=A\left(\zeta_{1}\right) / A_{0}$, as a function of $q_{0} h_{0}$. Results are 


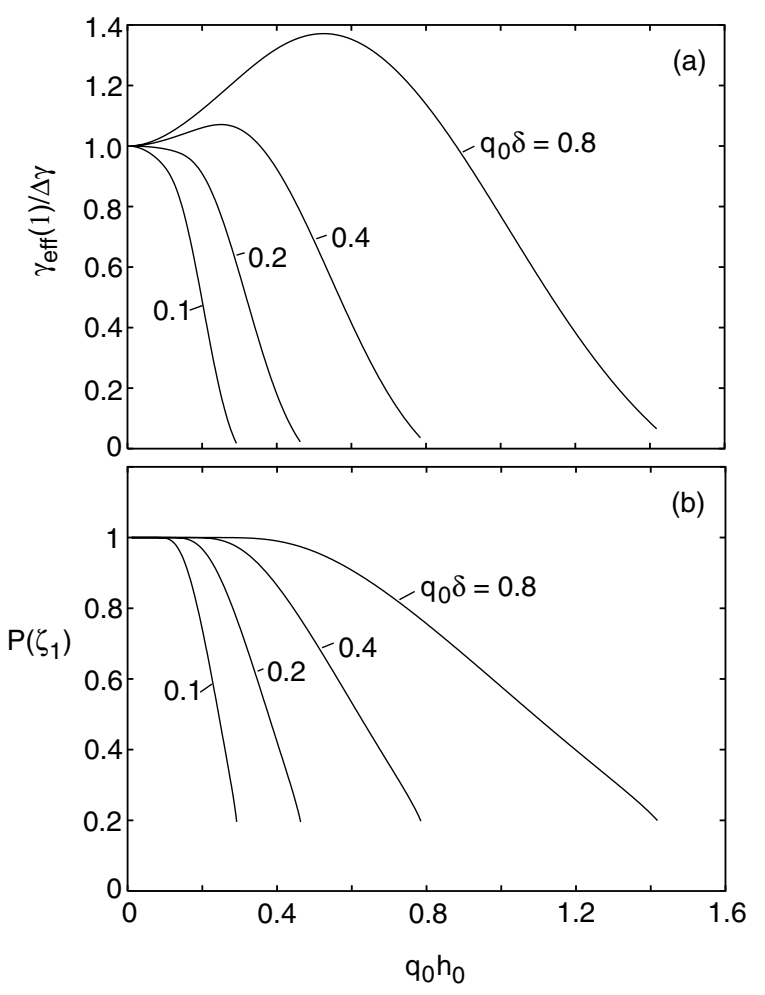

FIG. 2. (a) The macroscopic interfacial energy as a function of $q_{0} h_{0}$. (b) The normalized area of real contact, $P\left(\zeta_{1}\right)=$ $A\left(\zeta_{1}\right) / A_{0}$, as a function of $q_{0} h_{0}$. For $q_{0} \delta=0.1,0.2,0.4$, and 0.8 as indicated, and with $H=0.8$ and $\sigma_{0}=0$.

shown for $q_{0} \delta=0.1,0.2,0.4$, and 0.8 . We refer to $\gamma_{\mathrm{eff}}(1)$ at the magnification $\zeta=1$ as the macroscopic interfacial free energy which can be deduced from, e.g., the pulloff force for a ball using $F_{\mathrm{c}}=(3 \pi / 2) R_{0} \gamma_{\mathrm{eff}}(1)$. Note that for $q_{0} \delta=0.4$ and 0.8 the macroscopic interfacial energy first increases with increasing amplitude $h_{0}$ of the surface roughness (we define $h_{0}^{2}=2\left\langle h^{2}\right\rangle$ ) and then decreases. The increase in $\gamma_{\text {eff }}$ arises from the increase in the surface area. As shown in Fig. 2(b), for small $h_{0}$ the two solids are in complete contact, and, as expected, the complete contact remains to higher $h_{0}$ as $\delta \sim \Delta \gamma / E$ increases. Note also that the contact area is nonzero even when $\gamma_{\text {eff }}(1)$ is virtually zero: the fact that $\gamma_{\mathrm{eff}}(1)$ (nearly) vanishes does not imply that the contact area vanishes (even in the absence of an external load), but implies that the (positive) elastic energy stored at the interface just balances the (negative) adhesion energy from the area of real contact. The stored elastic energy at the interface is given back when the block is removed, and when $\gamma_{\text {eff }}(1) \approx 0$, it is just large enough to break the block-substrate bonding.

Figure 3 shows (a) the macroscopic interfacial energy and (b) the normalized area of real contact, $P\left(\zeta_{1}\right)=$ $A\left(\zeta_{1}\right) / A_{0}$, as a function of $q_{0} h_{0}$. The curves denoted by $a$ and $b$ correspond to the external pressure $\sigma_{0}=$ $0.05 E /\left(1-\nu^{2}\right)$ and $\sigma_{0}=0$, respectively. In 3(b) the dashed line is for no adhesion (i.e., $\Delta \gamma=0$ ) and for the same external pressure as for curve $a$. Note that, with increasing $h_{0}$, the area of real contact (when adhe-

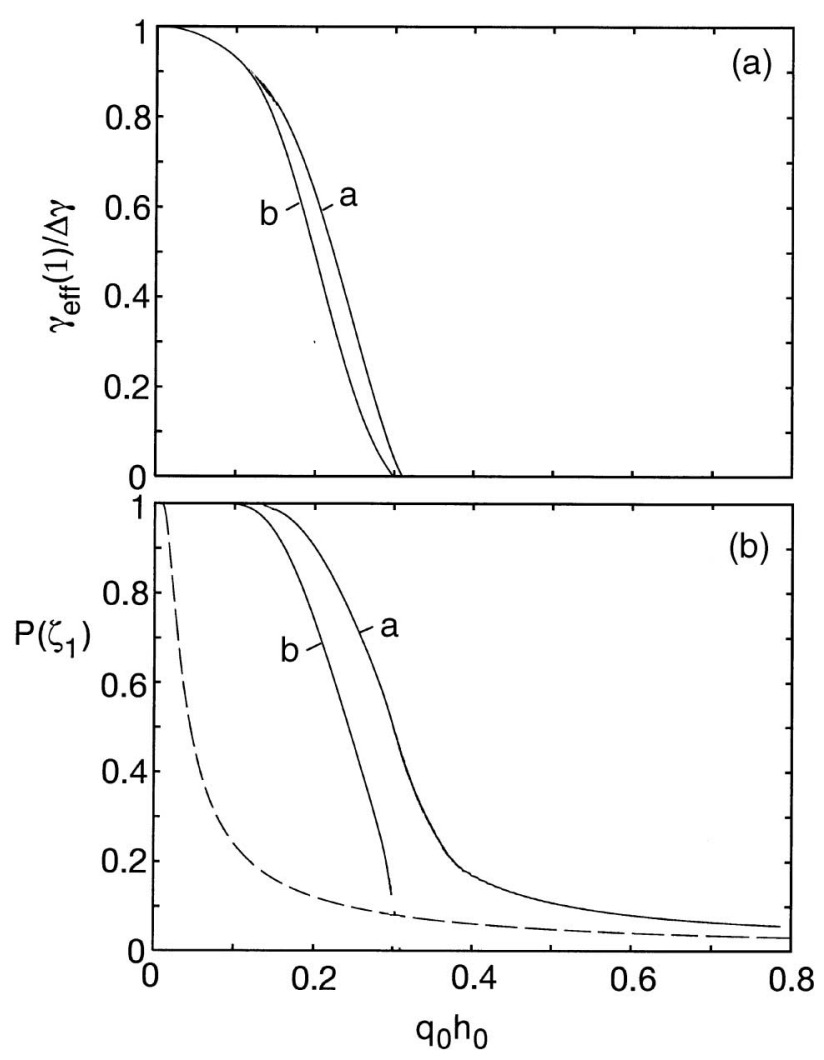

FIG. 3. (a) The macroscopic interfacial energy as a function of $q_{0} h_{0}$. (b) The normalized area of real contact, $P\left(\zeta_{1}\right)=$ $A\left(\zeta_{1}\right) / A_{0}$, as a function of $q_{0} h_{0}$. The curves denoted by $a$ and $b$ correspond to the external pressure $\sigma_{0}=0.05 E /\left(1-\nu^{2}\right)$ and $\sigma_{0}=0$, respectively. In (b) the dashed line is for no adhesion (i.e., $\Delta \gamma=0$ ) and for the same external pressure as for curve $a$. For $H=0.8$ and $q_{0} \delta=0.1$.

sion is included) only very slowly approaches the contact area that would result when the adhesion is neglected (dashed line). Since it is the area of real contact that is important in sliding friction, it is clear that the adhesion interaction may affect the friction force strongly even when no adhesion can be detected in a pulloff experiment; in the present case $\gamma_{\mathrm{eff}}(1)$ and, hence, the pulloff force vanish when $q_{0} h_{0}=0.3$. However, even when $q_{0} h_{0}=$ 0.5 , the area of real contact is more than twice as large when the adhesion is included as when it is neglected. The variation of $P(\zeta)$ and $\gamma_{\text {eff }}(\zeta)$ with the magnification $\zeta$ is shown in Fig. 4, for the same parameters as in Fig. 3 and for $q_{0} h_{0}=0.24$. Figure 4 (a) shows the dependence of $P(\zeta)$ on the magnification $\zeta$. Results are shown both with and without the adhesion interaction. Note that, without the adhesion, $P(\zeta)$ decreases monotonically with increasing magnification, and, in fact, without a short-distance cutoff, the area of real contact [corresponding to $P(\infty)$ ] vanishes. When adhesion is included, the (apparent) area of contact equals the area of real contact already at a rather small magnification $\zeta \approx 10$. This is only the case when the fractal dimension is close to 2 ; in Fig. 4 we have assumed $D_{\mathrm{f}}=2.2$. For a large 


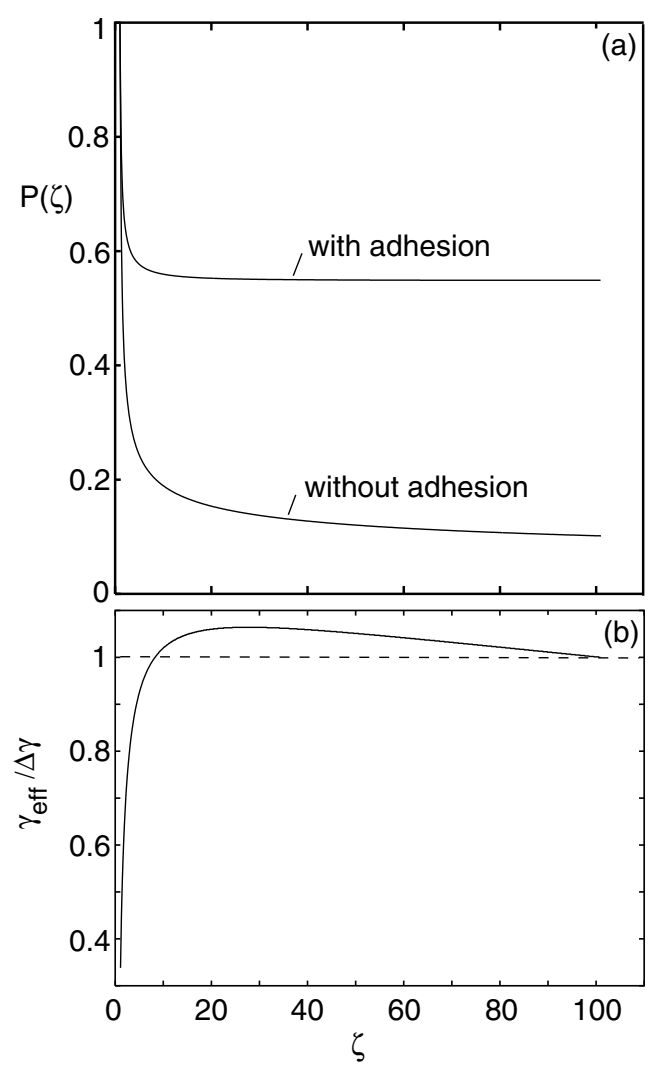

FIG. 4. The dependence of (a) the normalized (apparent) area of contact and (b) the effective interfacial energy, on the magnification $\zeta$. Results are shown with and without the adhesion interaction. $\zeta=1$ correspond to the long-distance cutoff length $\lambda_{0}$ in the fractal distribution. For the same parameters as in Fig. 3 and for $q_{0} h_{0}=0.24$.

fractal dimension, e.g., 2.6, the area of (apparent) contact decreases continuously with increasing magnification and, in fact, probably vanishes at infinite magnification (assuming no short-distance cutoff), in accordance with the qualitative discussion presented elsewhere [9].

Figure 4(b) shows the effective interfacial energy as a function of the magnification $\zeta$. Note that at short length scales (large $\zeta) \gamma_{\text {eff }}(\zeta)$ increases with decreasing magnification. This effect results from the increase in the surface area because of the surface roughness. However, at long length scales $\gamma_{\text {eff }}(\zeta)$ decreases below $\Delta \gamma$. This effect results from the contribution to the interfacial free energy from the elastic deformation energy induced in the rubber by the substrate surface roughness. Note also that $\gamma_{\text {eff }}$ at the shortest length scale (which in the present case corresponds to the magnification $\zeta=100$ ) equals the "bare" value $\Delta \gamma$ as it should.

The results presented in Fig. 2 are in good qualitative agreement with the experimental data of Fuller, Tabor,
Briggs, Briscoe, and Roberts [5-7]. Unfortunately, those authors did not measure the surface roughness power spectra, so no quantitative comparison is possible. We also note that, for rubber materials, the adhesion experiments must be performed extremely slowly in order to reduce the influence of viscoelastic effects [15].

To summarize, we have studied the influence of surface roughness on the adhesion of elastic solids. The theory allows for partial block-substrate contact on all length scales, which is particularly important for large fractal dimension [16].

[1] B. N. J. Persson, Sliding Friction: Physical Principles and Applications (Springer, Heidelberg, 2000), 2nd ed.

[2] J. N. Israelachvili, Intermolecular and Surface Forces (Academic, London, 1995).

[3] B. N. J. Persson, Phys. Rev. Lett. 87, 1161 (2001); J. Chem. Phys. 115, 3840 (2001); B. N. J. Persson, F. Bucher, and B. Chiaia, Phys. Rev. B 65, 184106 (2002).

[4] J. A. Greenwood and J. B. P. Williamson, Proc. R. Soc. London A 295, 300 (1966).

[5] K. N. G. Fuller and D. Tabor, Proc. R. Soc. London A 345, 327 (1975).

[6] G. A. D. Briggs and B. J. Briscoe, J. Phys. D 10, 2453 (1977).

[7] K. N. G. Fuller and A. D. Roberts, J. Phys. D 14, 221 (1981).

[8] C. Creton and L. Leibler, J. Polym. Sci., Pt. B: Polym. Phys. 34, 545 (1996); I. Chikina and C. Gay, Phys. Rev. Lett. 85, 4546 (2000).

[9] B. N. J. Persson and E. Tosatti, J. Chem. Phys. 115, 5597 (2001).

[10] K. L. Johnson, K. Kendall, and A. D. Roberts, Proc. R. Soc. London A 324, 301 (1971).

[11] See, e.g., L. B. Freund, Dynamics Fracture Mechanics (Cambridge University Press, New York, 1990).

[12] Here we have neglected the dependence of the surface energy $\gamma$ on the surface area during deformation. For the systems where the increase in the surface area is important, such as for rubber or gelatine, this is likely to be a good approximation.

[13] B. V. Derjaguin, Wear 128, 19 (1988); D. Tabor, in Surface Physics of Materials, edited by J. M. Blakely (Academic, New York, 1975), Vol. II, pp. 475-529.

[14] A self-affine fractal surface has the surface roughness power spectra $C(q) \sim q^{-2(H+1)}$. Since $C(q)$ is determined by $\langle h(\mathbf{x}) h(\mathbf{0})\rangle$, the higher order correlation functions can take any form. In particular, the height probability distribution $P_{h}=\langle\delta(h-h(\mathbf{x})\rangle$ can be a Gaussian, as is often observed experimentally.

[15] M. Barquins and D. Maugis, J. Adhes. 13, 53 (1981).

[16] B. N. J. Persson, Eur. Phys. J. E 8, 385 (2002). 